\title{
Fatigue behaviour of an industrial synthetic rubber
}

\author{
Thomas Balutch ${ }^{1,3}$, Bertrand Huneau ${ }^{1, *}$, Yann $\mathrm{Marco}^{2}$, Pierre Charrier ${ }^{3}$, and Clément Champy $^{3}$ \\ ${ }^{1}$ Institut de Recherche en Génie Civil et Mécanique (GeM), UMR CNRS 6183, Ecole Centrale de Nantes, 44300 Nantes, France \\ ${ }^{2}$ Institut de Recherche Dupuy de Lôme (IRDL), FRE CNRS 3744, ENSTA Bretagne, 29200 Brest, France \\ ${ }^{3}$ Vibracoustic Nantes, CAE Durability Department, 1 Rue du Tertre, 44470, Carquefou, France
}

\begin{abstract}
For some automotive anti-vibration applications, for instance exhaust hangers, center bearing bushes or torsional vibration dampers, temperature constraints make the use of synthetic rubbers, such as EPDM, necessary because of their better heat aging resistance compared to natural rubber. The aim of this paper is to understand the features of the fatigue behaviour of an industrial EPDM compared to the wellknown natural rubber. To do so, fatigue tests are conducted on hourglass-shaped specimens, and fracture surfaces are analysed using optical microscopy and scanning electron microscopy (SEM). It appears that every samples exhibit only one root cause of failure. Thus, two types of precursors are identified as responsible of the final fracture of samples: material's inclusions and mold flaws. Interrupted fatigue tests are then performed and fatigued samples are observed with SEM. The built procedure allows us to follow fatigue cracks initiation and propagation along cycles, and to propose local damage mechanisms for each type of precursors. A global damage scenario is finally considered and compared to the one of natural rubber described in the literature.
\end{abstract}

\section{Introduction}

Anti-vibration solutions in automotive are crucial to ensure comfort, safety and durability in vehicle systems. Most of the parts that are designed in Vibracoustic to provide these functions are made of rubber, and particularly of natural rubber (NR), known for its good fatigue resistance that is often attributed to its faculty to crystallize under strain [1]. Because of the significant heat experienced by some parts near car's hot-spots, natural rubber can no longer be used (long periods with $\mathrm{T}>90^{\circ} \mathrm{C}$ ). As a consequence, synthetic rubbers such as EPDM are preferred because of better heat aging resistance.

However, fatigue prediction for this material is difficult due to an important scattering of the lifetime results that can reach more than 4 decades (see Fig. 1).

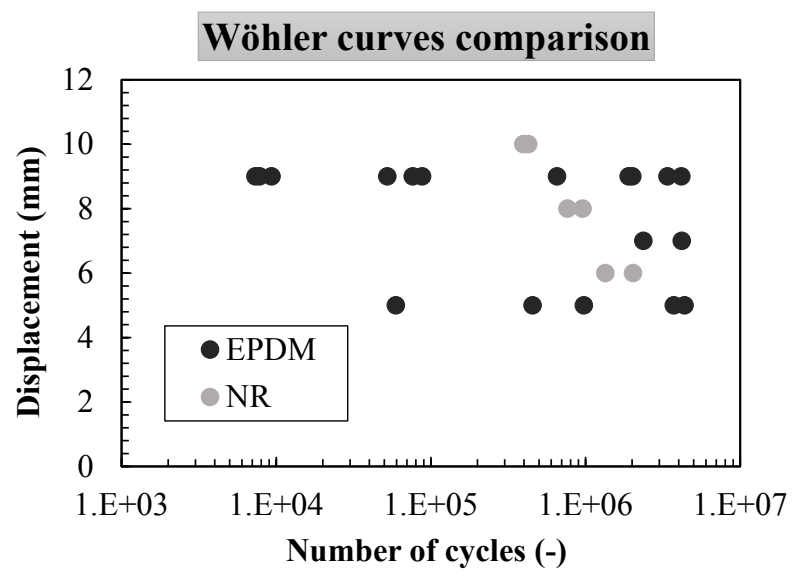

Fig. 1. Fatigue results scattering of EPDM compared to natural rubber (data from Flamm et al. [2]).

Moreover, this rubber matrix presents little or no strain-induced crystallization which leads to a lower resistance to fatigue crack growth when compared to natural rubber [3-5]. Finally, it has to be underlined that compared to natural rubber, only few scientific papers are dealing with the fatigue behaviour of EPDM [2-10].

Thus, the aim of this paper is to highlight the specificities of the fatigue behaviour of an EPDM, and to compare them with the ones of the more known natural rubber.

\section{Experiments}




\subsection{Material and samples}

The material of this study is a fully formulated industrial EPDM, filled with carbon black and crosslinked with sulfur. As it is used for real mass-produced parts, the quantitative results will not be provided.

The samples used to perform the mechanical tests are known as AE2 samples [11]. It is an hourglass-shaped specimen with a notch radius of $2 \mathrm{~mm}$. The important strain gradient induced by this geometry focuses fatigue crack initiations close to the skin of the median area. On top of that, to be representative of real parts' process, injection molding is used to manufacture this specimen. As a consequence, all AE2 present a specific mold flaw on the zone that corresponds to the junction of the two parts of the mold (see Fig. 2).

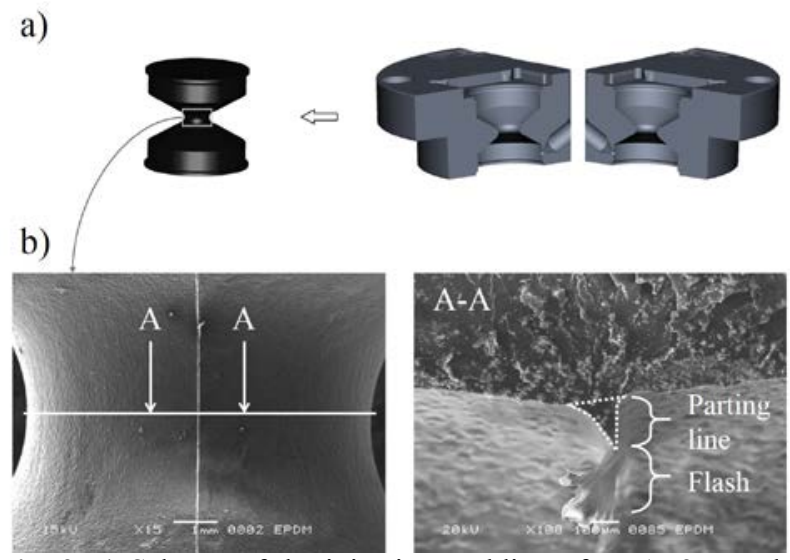

Fig. 2. a) Scheme of the injection molding of an AE2 sample and b) SEM observations of its related mold flaws.

\subsection{Observations tools}

In order to analyse fracture surfaces and fatigue cracks on the surface of AE2 samples, two different observations techniques are used.

The first one is stereo microscopy. The stereo microscope Carl Zeiss STEMI 2000C available in Vibracoustic can take numerical pictures with magnification from $\times 6.5$ to $\times 50$.

The second one is scanning electron microscopy (SEM). Two microscopes are used in this study. A JEOL 6060-LA with an EDS (Energy Dispersive Spectroscopy) probe to perform quantitative chemical analysis. It is located at "Ecole Centrale de Nantes" and used for fracture surfaces analysis. The other one is a JEOL JCM 6000plus used for interrupted fatigue tests analysis at Vibracoustic. As the scale of interest for this study goes from some micrometres to several millimetres, the highest magnification reached here is approximately x 2000 .

Thanks to carbon black, the rubber is sufficiently conductive to use secondary electrons in high vacuum without using any metal deposit. Depending on the test, SEM parameters have to be set accordingly. For fracture surfaces analysis, the acceleration voltage is set to $20 \mathrm{kV}$ with a medium spot size in order to have a good quantitative EDS analysis. For interrupted fatigue tests, the energy of the electron beam must be reduced in order not to damage the rubber, which could influence the fatigue life and the related damage scenario. To overcome this difficulty, the acceleration voltage is set to $10 \mathrm{kV}$, the spot size is small, the magnification for pictures remains low, and a special attention is given to the exposure time in the SEM. The samples are stretched of $3 \mathrm{~mm}$ when put in the SEM, in order to open the cracks.

\subsection{Fatigue tests}

The fatigue tests are conducted on a hydraulic bench in which 5 samples can be set up at once. The mechanical load is displacement controlled, and the frequencies are adapted for each load level in order to limit the selfheating of the rubber however keeping a correct amount of experimental time. Only relaxing conditions are tested in this study $(\mathrm{R}=0)$, at $25^{\circ} \mathrm{C}$. Higher testing temperature is for sure a perspective of the study.

First, a Wöhler curve is built with five load levels, and five samples per load levels. After that, an additional test internally called "radar test" is conducted. This test consists in breaking 25 samples at the same load level to better estimate the scattering of the lifetime results.

The loss of effective stiffness is used as the end of life criterion (see Ostoja-Kuczynski et al. [11] for more details). The end of life is defined as the detected initiation $\left(N_{\mathrm{i}}\right.$ number of cycles, see Fig. 3), and corresponds approximately to the presence of a $2 \mathrm{~mm}$ long crack on AE2 samples. This criterion has the advantages of being easy to use with automated posttreatment, and more representative of the failure of real parts, that will lose their mechanical properties before total fracture. As a fatigue parameter, the maximum principal strain in the notched area is used to plot all the fatigue life results.

\subsection{Interrupted fatigue tests}

The purpose of these tests is to analyse the initiated cracks on samples that have undergone some fatigue cycles, before total failure. In the literature, several authors built protocols for different rubbers [12-15]. Because of the important scattering of the fatigue results, and the fast fatigue crack propagation in EPDM, it is not possible to use the same protocol as Le Cam et al. [12] and to wait for a crack of a certain size. Indeed, the step between no visible cracks and total failure is too short and easy to miss. Moreover, the use of a certain percentage of the mean duration life for different specimens cannot be considered as in Huneau et al. [15]. Samples could be completely broken or not damaged at all after fatigue testing and this would make the analysis irrelevant. Therefore, the same samples need to be monitored along fatigue cycles in order to be able to link the damage of each sample to its own duration life.

In consequence, 10 samples are chosen to be representative of the scattering of the results. SEM pictures of the full median side surface are taken and specimens are then subjected to $X$ number of cycles. These 2 steps are repeated until complete fracture of all 
the samples. The load level is the one of the "radar test", because it is where the material's fatigue behaviour is the best described. As mentioned in section 2.2, the electron beam can have an influence on the fatigue behaviour, so a special care is given to the acquisition of SEM pictures. This is also why the use of repeated tomography measurements has been rejected, considering the needed long exposure time and high energetic x-rays beam for one complete scan.

At last, the number of cycles $X$ needs to be correctly chosen, to be sure not to miss the propagation of a main crack. In Fig. 3 is plotted the maximum force versus the number of cycles curve, which we use to defined $X$ thanks to equations (1) and (2).

$$
\begin{gathered}
N_{\mathrm{p}}=N_{\mathrm{f}}-N_{\mathrm{i}} \\
X=N_{\mathrm{p}} / 2
\end{gathered}
$$

With $N_{\mathrm{f}}$ the number of cycles until failure, $N_{\mathrm{i}}$ the number of cycles until the detection of initiation, and $N_{\mathrm{p}}$ the number of cycles of macroscopic propagation. This protocol is justified by the fact that $N_{\mathrm{p}}$ is almost not scattered compared to $N_{\mathrm{i}}$ as it is shown later on Fig. 7.

The worst possible case would be that no cracks initiations are visible on the surface before $N_{\mathrm{i}}$ number of cycles, which is possible because of the important crack growth rate of EPDM. By relating $X$ to half of the macroscopic propagation, at least one observation of the main crack before total failure is possible.

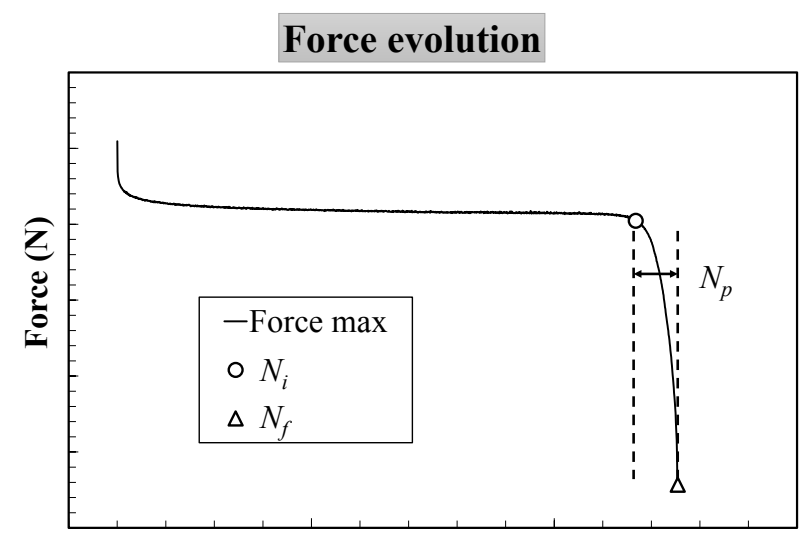

Number of cycles (-)

Fig. 3. Maximum force evolution of an AE2 sample for "radar test" load level versus the number of cycles.

\section{Results and discussion}

\subsection{Fractographic analysis}

Once a sample is totally broken, it is possible to find the root causes of failure by analysing its fracture surface, which is very different depending on the material. The fracture surfaces of the studied EPDM and a natural rubber from another internal work are presented in Fig. 4.

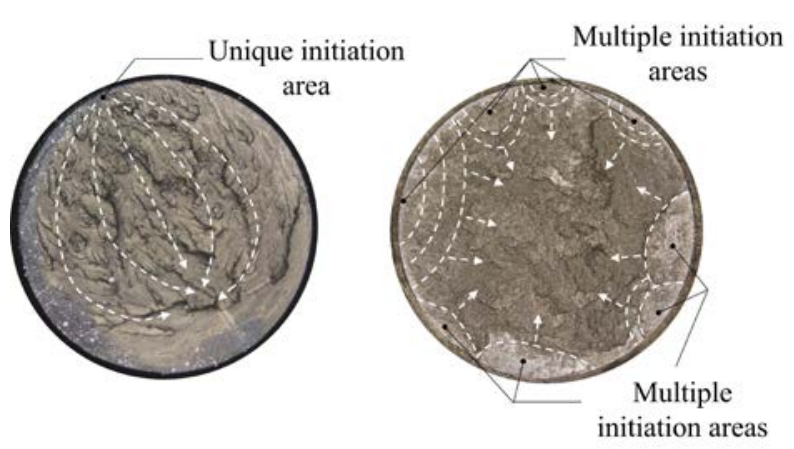

Fig. 4. Fracture surfaces analysis of an EPDM (left) and a natural rubber (right) with stereo microscopy.

For natural rubber, the surface appears very rough, and semi-circular arcs, which can be linked to initiation sites, are highlighted all around the skin. The final failure occurs at the centre of the fracture surface. Thus, the fatigue life seems to be governed by multiple cracks initiation and fatigue cracks coalescence.

For EPDM, the fracture surface appears different. The smooth part in the bottom right corresponds to the final "brittle" fracture. The rest of the surface is associated with fatigue "rivers" that converge to one spot only, that is the single root cause of failure. The specific roughness decreases when approaching the root cause. At the end, every EPDM's fracture surfaces exhibit only one root cause of failure.

When a focus is made on the initiation spot, it is possible to split precursors in two categories: inclusions, and parting line region. Inclusions are agglomerates from ingredients of the rubber's formulation and parting line region is the mold flaw presented in Fig. 2, including the parting line and the flash. This split is made because the physical origin of these two flaws are very different: one is intrinsic to the material, and the other is purely geometrical, and only process dependent.

The initiation spot is easy to find with stereo microscopy, but SEM is needed to check the presence of inclusion and to analyse it with EDS. Moreover, SEM enables us to confirm that parting line region is really responsible of initiation and not an inclusion close to this region. Fig. 5 shows an example of an inclusion initiating a fatigue crack. Thanks to all SEM pictures, it is also possible to measure the dimensions of the precursors. The geometrical parameter used to represent the global precursor's size is $\sqrt{\text { Area }}$. It has been initially proposed by Murakami and Endo [16] for fatigue of metals, and is still used nowadays, for example in references $[17,18]$.

However, an inclusion is not systematically found for initiation outside the parting line region. One explanation for this can be that the inclusion responsible of the failure fell from the fracture surface. Another explanation can be that an inclusion is below the surface, covered with rubber, that makes it non visible with surface observations like SEM. 


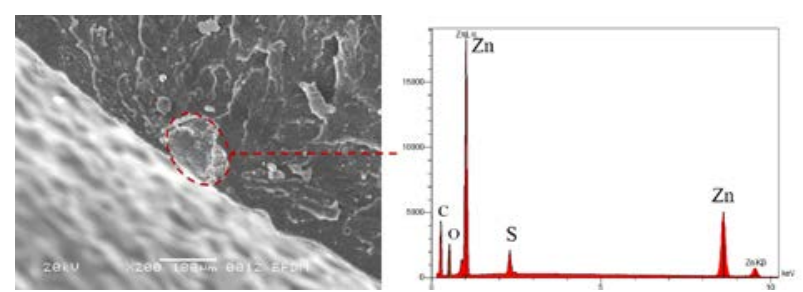

Fig. 5. SEM observation of a zinc oxide agglomerate initiating a crack (left) and its related EDS analysis (right).

\subsection{Lifetime results}

Fatigue tests are conducted according to section 2.3 and all the lifetime results are plotted on Fig. 6. To keep reasonable test durations, some tests have been interrupted before total failure, and are represented with an arrow on the right. As expected, there is an important scattering (more than 2 decades), yet the mean duration lives of every load level are aligned well enough to fit a correct power law.

$N_{\mathrm{p}}$ is measured for every sample, and Fig. 7 shows the evolution of this number against the load level. It appears that there is very little scattering on this number compared to $N_{\mathrm{i}}$ on Fig. 6. This suggests that the scattering of the fatigue results comes rather from the initiation step than the propagation one.

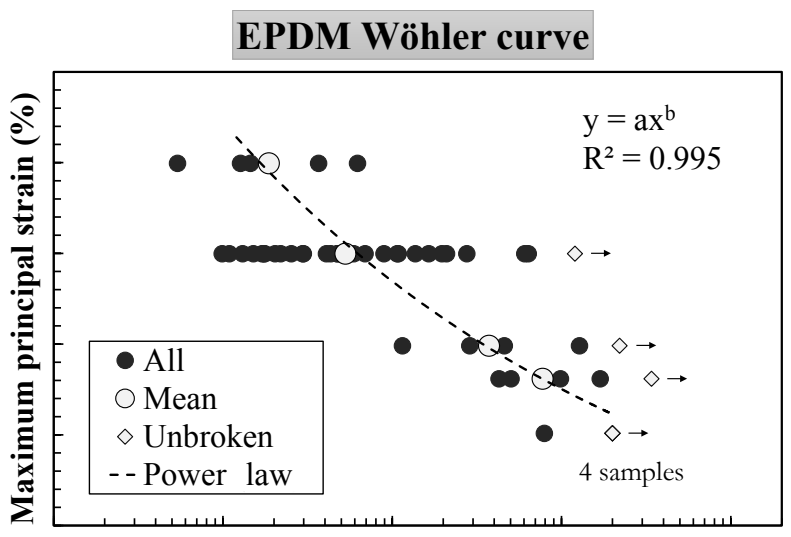

Number of cycles to initiation (-)

Fig. 6. Wöhler curve of the studied EPDM including "fatigue radar" data.

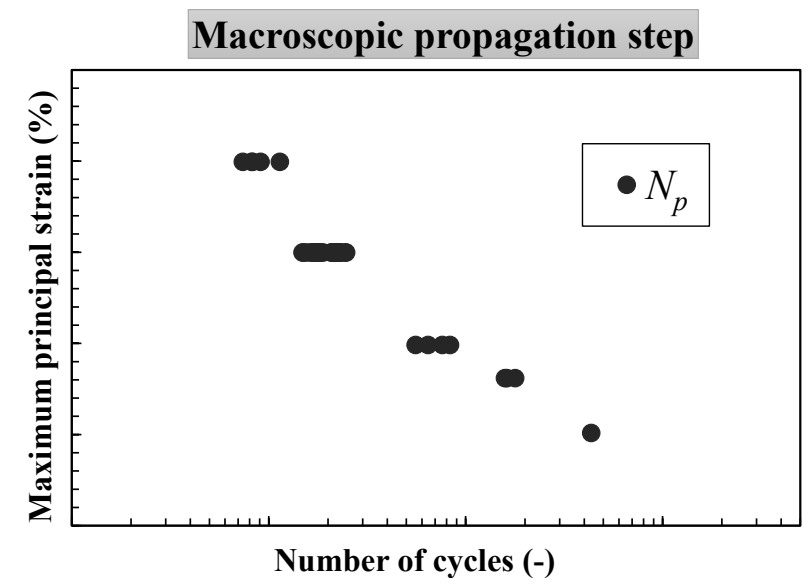

Fig. 7. Number of cycles for macroscopic propagation.

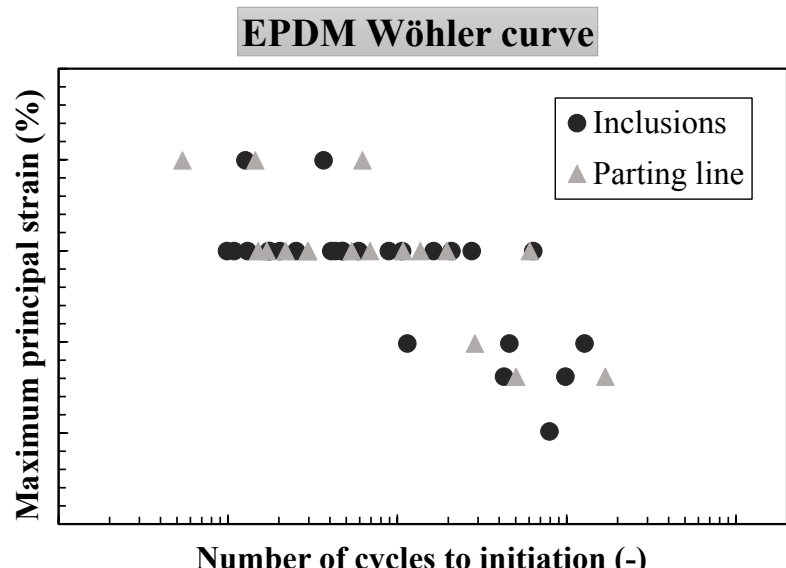

Fig. 8. Wöhler curve with a split by type of precursors.

Thanks to the fractographic analysis described in section 3.1, the fatigue results can be split by type of precursors on Fig. 8: inclusions or parting line region. It seems that there is no real tendency on the fatigue results between initiation on purely geometrical flaws that are process dependent, and on natural intrinsic flaws. Globally, $60 \%$ of failures occur on inclusions, and $40 \%$ on parting line. This means that the geometry of the parting line region is almost as critical as the inclusions present in the material. This results is only valid for the mold geometry considered here, and could be different for another sample geometry, or even for AE2 manufactured with a different mold.

On Fig. 9, the influence of the size of inclusions versus the number of cycles is plotted, considering only the "radar test" load level. Fig. 9 reveals that there is no direct correlation between the size of an inclusion and the duration life. The scattering of the fatigue results cannot be explained only by a scattering in the size of inclusions, and other key parameters must be considered, as the chemical composition, the exact location of the inclusion in the sample, or its 3D shape.

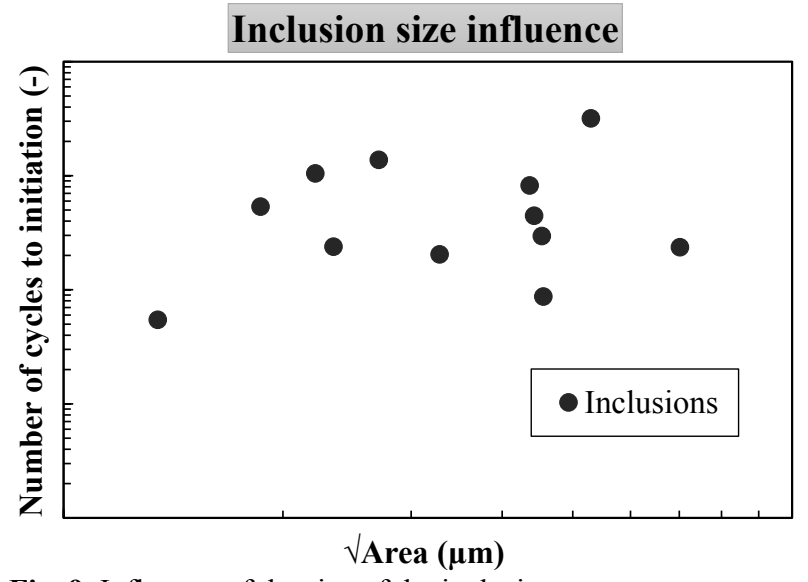

Fig. 9. Influence of the size of the inclusions. 


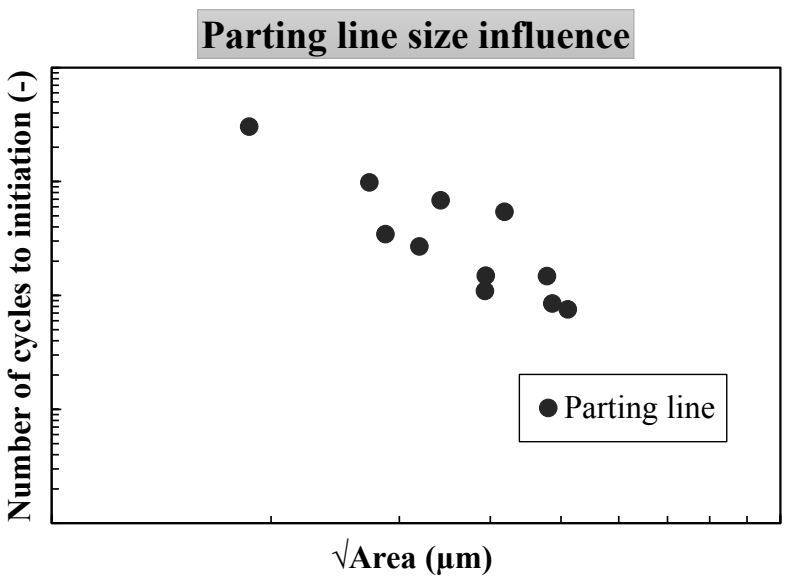

Fig. 10. Influence of the size of the parting line.

Conversely, the same thing has been done for parting line by taking the area defined by the dashed line in Fig. 2. The results in Fig. 10 show a good correlation between the size of the parting line and the duration life. It appears that the duration life decreases with an increasing size of the parting line. As a consequence, the scattering of the fatigue results for initiation on parting line region can be explained by the one of the parting line geometry. This result proves that small fluctuations of the precursor's geometry can have a huge influence on the lifetime results. In fact, the parting line geometry is assumed to be highly dependent on all the process parameters, and could be very difficult to control.

Finally, it has to be noted that a split according to the chemical nature of the inclusions could not have been made, except for zinc oxide agglomerates. For the rest of the inclusions, EDS spectrum were very similar and presented not enough differences to build categories.

\subsection{Damage scenario}

\subsubsection{Crack tip}

Thanks to interrupted fatigue tests, the crack tip is pictured along fatigue cycles. Fig. 11 shows that the crack propagation mechanisms are associated with ligaments. Similar crack tip has been observed for natural rubber, silicone rubber and EPDM in references [19-21]. These ligaments break under tension and cause the roughness of the fracture surface. It also appears on Fig. 11 that the dimensions of the ligaments increase when the crack grow, which is in accordance to an increasing roughness on the fracture surface (see section 3.1). Le Cam et al. [22] also noticed this evolution of the roughness on fracture surfaces of natural rubber.
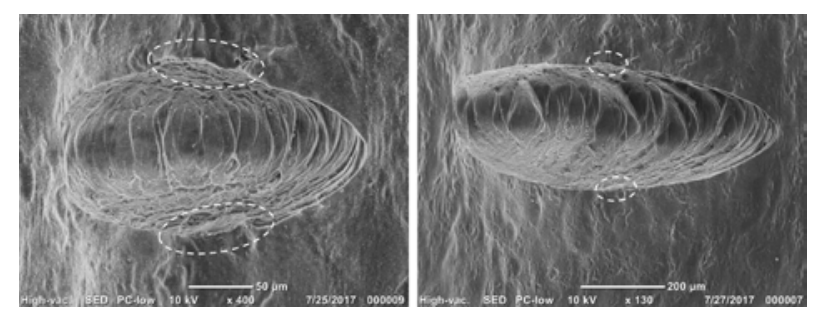

Fig. 11. Crack tip shape evolution before (left, $\mathrm{x} 400$ ) and after (right, x130) some fatigue cycles.

\subsubsection{Crack initiation on inclusions}

For crack initiation on inclusions, two different mechanisms have been observed. They will be referred as mechanism A and B for the rest of the article.

Mechanism A is related to inclusions that have high internal cohesion compared to the bonding with the matrix and can be described as follow (see Fig. 12):

- Stage 1: debonding at one pole;

- Stage 2: opening on the sides;

- Stage 3: propagation into the bulk.

Mechanism B is related to inclusions exhibiting low internal cohesion and is defined by (see Fig. 13):

- Stage 1: inclusion's fracture;

- $\quad$ Stage 2: opening on the sides;

- $\quad$ Stage 3: propagation into the bulk.
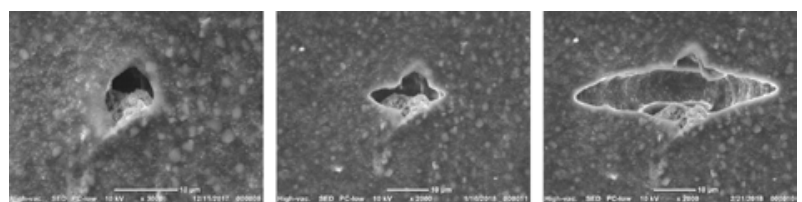

Fig. 12. Damage mechanism A, stage 1, 2, 3 from left to right.
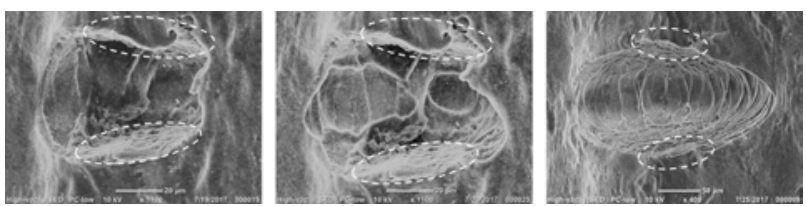

Fig. 13. Damage mechanism $B$, stage 1, 2, 3 from left to right.

The internal cohesion and interface with the matrix is assumed to depend on the type and size of the inclusion, as well as on the rubber matrix. In this study, cracks initiate from zinc oxide agglomerates following mechanism A, and following mechanism B for all other inclusions. These two mechanisms are schematized on Fig. 14. The main difference between these two mechanisms is the stage 1 . Stage 3 corresponds to a "long" crack that is no longer influenced by its precursor. 


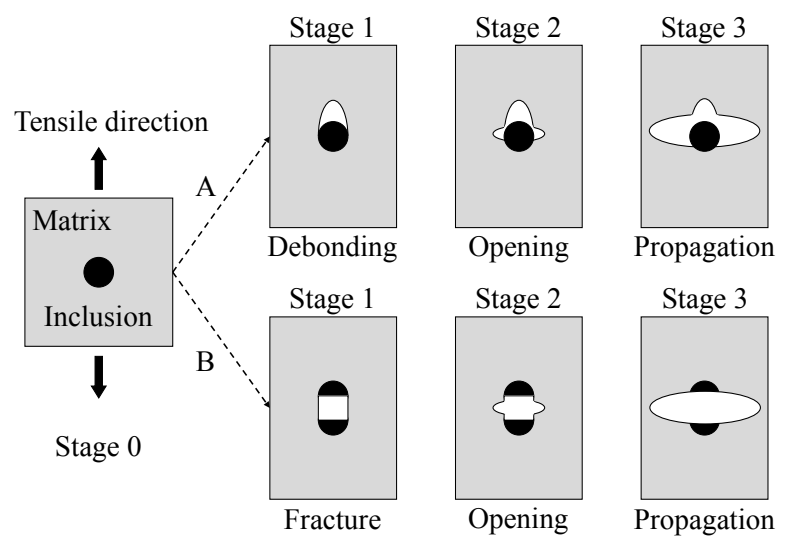

Fig. 14. Scheme of the initiation mechanisms on inclusions.

For natural rubber, Huneau et al. [15] have also proposed these two initiations mechanisms. Mechanism A was observed for crack initiation on rigid inclusions such as carbon black agglomerates (from $9 \mu \mathrm{m}$ to $17 \mu \mathrm{m}$ ), and mechanism B for small zinc oxide agglomerates (average size of $7 \mu \mathrm{m}$ ), but most of the time, the authors only witnessed the stage 1 of mechanism B. Perhaps they would have seen the stages 2 and 3 more often, if their zinc oxide agglomerates were bigger than $10 \mu \mathrm{m}$, as it is the case for the inclusions following mechanism $\mathrm{B}$ in this study. Furthermore, the fact that 2 different damage mechanisms (A for EPDM, and B for natural rubber) are observed for inclusions of the same type (zinc oxide agglomerates) in different rubber matrix, proves that the balance between internal cohesion and inclusion/matrix interface properties could be very complex to evaluate.

To sum-up, it appears that the considered local damage mechanisms are similar for EPDM and natural rubber, but that the correspondence between these mechanisms and the inclusions initiating cracks is not the same.

\subsubsection{Crack initiation on the parting line}

Fatigue crack initiation related to the parting line region has been followed for every sample with a special care, because it is known from the fractographic study that it can be critical for the fatigue behaviour of this EPDM. The proposed damage scenario is presented on Fig. 15 and can be described as:

- Stage 1: tearing of the flash;

- Stage 2: propagation into the parting line;

- Stage 3: propagation into the bulk.

Thanks to all observations, it is assume that stage 1 is mostly dependent on the flash's geometry. The step between stage 2 and stage 3 appears to be more dependent on the parting line geometry. This last step could be the critical one, because once a crack is in the bulk, it propagates until the final failure. When a crack initiates on the flash, the parting line could act like the last barrier preventing the crack propagation into the bulk. Thus, the fatigue failure would be more dependent on the parting line features than on the flash ones, and these observations could confirm the good correlation between the size of the parting and the fatigue results in Fig. 10.

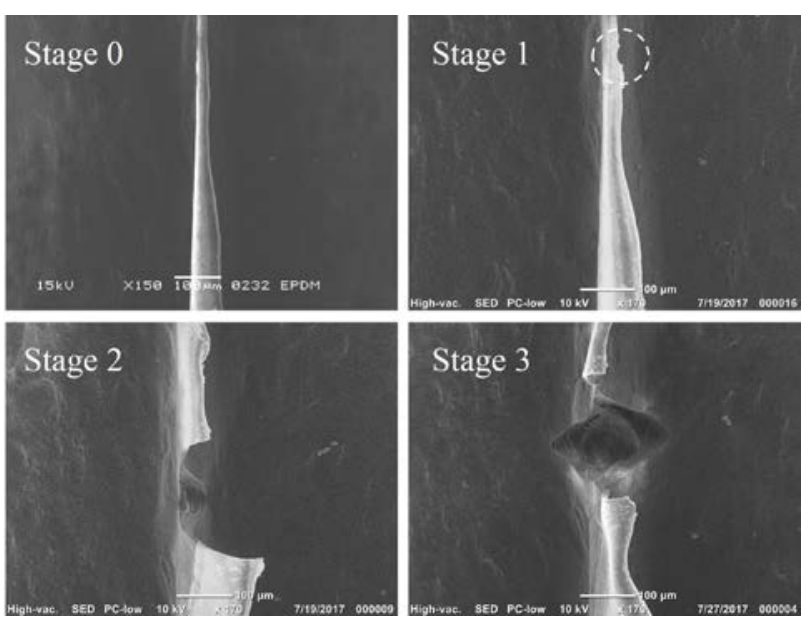

Fig. 15. Scheme of the initiation mechanisms on inclusions.

Finally, a two steps initiation mechanism can also be considered. As the parting line region is created by a flow of rubber between the two parts of the mold, an inclusion can be stuck in the parting line region by a funnel effect. This creates a high stress/strain concentration that eventually initiates a crack in the parting line region, but due to an inclusion.

These mechanisms have been observed for natural rubber [23], even if not described in details. The initiation mechanisms on the parting line therefore seem very similar for EPDM and natural rubber.

\subsubsection{Competition between fatigue cracks}

Fig. 16 illustrates that over a whole AE2 sample, several stage 3 cracks can be observed at the same time. These long cracks, initiated from inclusions and in the parting line region, are quiet distant so that they do not influence each other. For every tested sample, one of these initiated cracks finally takes the lead and propagates until the complete fracture of the specimen, without any cracks coalescence. This confirms the fractographic analysis in section 3.1 where only one root cause of failure is found. As shown on Fig. 16, stage 3 cracks were seen only belatedly compared to the duration life of the considered sample. 
a)

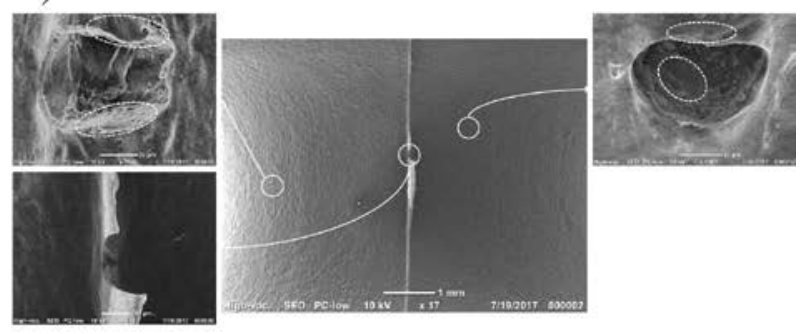

b)

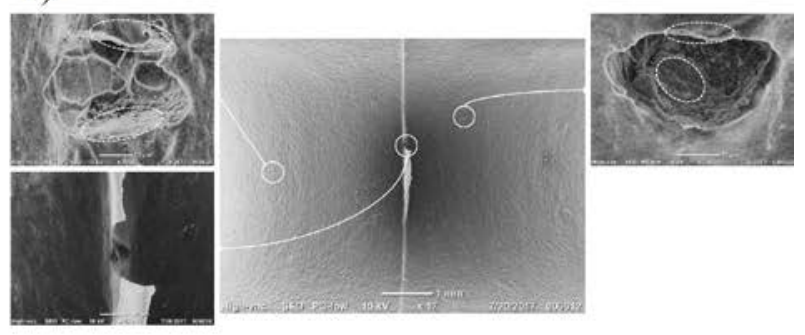

c)

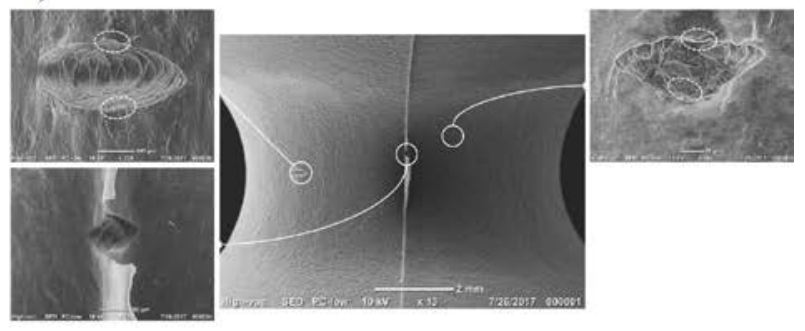

d)

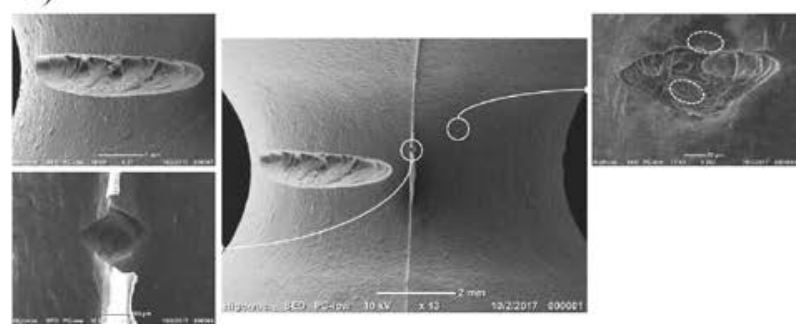

Fig. 16. Fatigue cracks evolution in EPDM at different duration lives, and different magnifications: a) $30 \%$ of the total duration life, b) $40 \%$, c) $85 \%$, d) $100 \%$.

Overall, the considered damage scenario is the following:

- Few stage 1 initiations both on inclusions and in the parting line region;

- Some stage 2 and stage 3 cracks, but far from each other and without fatigue cracks coalescence. This step occurs belatedly during the fatigue life of the sample;
- One of the cracks eventually propagates until final fracture of the specimen.

Thus, the fatigue failure of EPDM samples seems to be driven only by the initiation of a crack on a "critical" flaw, considering the mechanical load. The scattering of the fatigue results could be explained by the fluctuation of this flaw from one sample to the other. In fact, for material's inclusions, not only the type and shape but also the precise location in the sample could have an important influence. For instance, a big inclusion far from the skin will be harmless compared to a smaller one stuck into the parting line. Moreover, one can imagine that the geometry of the parting line region is highly dependent not only on every parameter of the process, but also on how the sample is removed from the mold, or manipulated after removal. All of these small variations could create an important scattering in the fatigue results.

Considering the literature of the fatigue behaviour of natural rubber $[12-15,21-25]$, the global damage scenario is very different. Indeed, for a natural rubber, cracks are far more numerous (see Fig. 17). Furthermore, it appears that they can initiate during the first fatigue cycles, and this step is followed by a slow crack propagation. Interrupted fatigue tests, as well as fracture surfaces analysis show that cracks coalescence finally causes the final fracture of natural rubber specimens (see Fig. 4). Thus, even if a harmful inclusion is present close to the skin of the median area, it will not be the only root cause of failure.

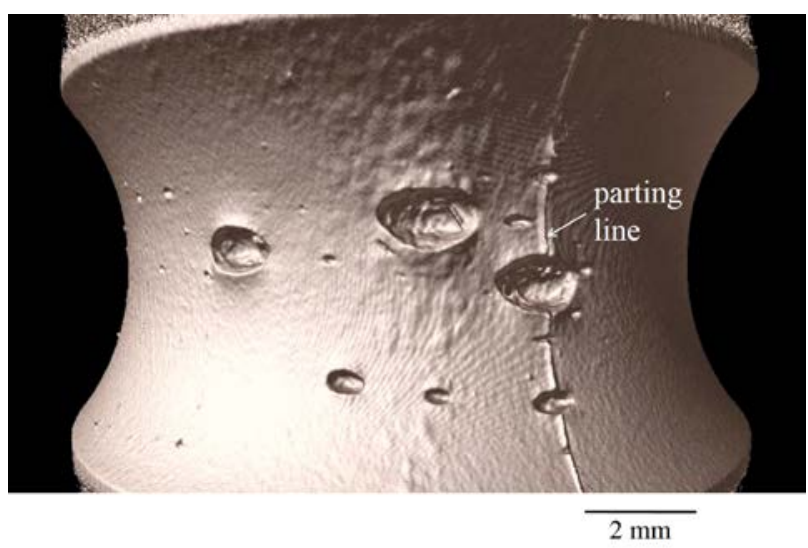

Fig. 17. 3D view of cracks in a natural rubber after 100000 fatigue cycles, from microtomography, by Huneau et al. [25].

As a results, fatigue failure of natural rubber is driven by an early multi-initiation, a multi-propagation, and cracks coalescence, whereas for EPDM, only one critical flaw which exhibits a late initiation is responsible of the final fracture. Accordingly, the propagation step, which is known to be little scattered, represent an important part of the total lifetime of natural rubber. Conversely, as EPDM presents an elevated fatigue crack growth rate [35], the initiation step seems to be the key to understand its fatigue behaviour. These main differences between the two considered rubbers could explain why there is so little scattering in the fatigue results of natural rubber compared to EPDM. 
At last, it has to be noted that the fatigue lifetime results are highly influenced by the chosen sample's geometry.

\section{Conclusion}

The important scattering of the EPDM fatigue results is confirmed by the build of a Wöhler curve with AE2 samples. It appears that the macroscopic propagation is almost not scattered, and this suggests that the scattering of the lifetime results comes from initiation step rather than propagation one. The related fracture surface exhibit only one root cause of failure that can be either inclusions of the rubber's formulation, or purely geometrical flaws created by the process. Approximately $60 \%$ of the failure occurs on inclusions, and $40 \%$ on parting line region but without any clear influence on the lifetime results. It has to be noted that this result is highly dependent on the mold, and sample geometry. A good correlation appears between the $\sqrt{\text { Area }}$ of the parting line and the fatigue life results, so the scattering in the fatigue failure could be explained by a fluctuation in the parting line geometry. However, no tendencies are noticed on the fatigue results for the $\sqrt{\text { Area }}$ of inclusions. This could be explained by the important influence of other key parameters as the nature of the inclusion, its precise 3D shape, or its precise location, for instance the distance to the median zone, and the distance to the skin.

Thanks to interrupted fatigue tests with regular SEM observations, local damage mechanisms are proposed for each type of precursors. It appears that they are very similar to what has been proposed for natural rubber $[15$, 23]. Nevertheless, the main differences come from the global damage scenario. For natural rubber, it has been shown [12-15, 21-25] that an important number of cracks initiate during the first fatigue cycles. This multiinitiation followed by a slow propagation and cracks coalescence could explain the low scattering of the fatigue results of natural rubber. On the opposite, for the studied EPDM, only a few number of cracks initiate far from each other, and one of them eventually propagates until complete fracture of the specimen without any cracks coalescence. Thus the fatigue failure of a given sample seems to be driven only by its most "critical" flaw regarding fatigue, and this could explain the important scattering in the fatigue results compared to natural rubber. Furthermore, the fact that stage 3 cracks, whatever the origin of the precursor, occurs only belatedly in the duration life of the sample, proves that the total lifetime cannot be reduced to crack propagation of initial flaw, and this needs further investigations.

\section{References}

1. W. Mars, A. Fatemi, Rubber Chem. Technol. 77, 391-412 (2004)

2. M. Flamm, T. Steinweger, J. Spreckels, T. Brüger, ECCMR V, 233-238 (2008)
3. F. Abraham, G. Clauß, T. Alshuth, J. Kroll, Kaut. Gummi Kunstst. 58, 595-599 (2005)

4. W. W. Chung, Y. W. Chang, Korea Polym. J. 9, 319-326 (2001)

5. J. Royo, Polym. Test. 11, 325-344 (1992)

6. F. Abraham, T. Alshuth, S. Jerrams, Mater. Design 26, 239-245 (2005)

7. H. W. Chou, J. S. Huang, S. T. Lin, J. Appl. Polym. Sci. 103, 1244-1251 (2007)

8. C. Gögelein, H. J. H. Beelen, M. Van Duin, Soft Matter 13, 4241-4251 (2017)

9. H. Ismail, N. S. Che Mat, N. Othman, J. Vinyl Addit. Techn. 31, 5-12 (2017)

10. S. Jerrams, J. Hanley, N. Murphy, H. Ali, Rubber Chem. Technol. 81, 638-649 (2008)

11. E. Ostoja-Kuczynski, P. Charrier, E. Verron, G. Marckmann, L. Gornet, G. Chagnon, ECCMR III, 41-47 (2003)

12. J. B. Le Cam, B. Huneau, E. Verron, L. Gornet, Macromolecules 37, 5011-5017 (2004)

13. V. Le Saux, Y. Marco, S. Calloch, P. Charrier, Polym. Eng. Sci. 51, 1253-1263 (2011)

14. K. Legorju Jago, Rubber Chem. Technol. 85, $387-$ 407 (2012)

15. B. Huneau, I. Masquelier, Y. Marco, V. Le Saux, S. Noizet, C. Schiel, P. Charrier, Rubber Chem. Technol. 89, 126-141 (2016)

16. Y. Murakami, M. Endo, Eng. Fract. Mech. 17, 1-15 (1983)

17. M. I. Houria, Y. Nadot, R. Fathallah, M. Roy, D. M. Maijer, Int. J. Fatigue 80, 90-102 (2015)

18. V.D. Le, F. Morel, D. Bellett, N. Saintier, P. Osmond, Mat. Sci. Eng. A-Struct. 649, 426-440 (2016)

19. S. Beurrot, B. Huneau, E. Verron, J. Appl. Polym. Sci. 117, 1260-1269 (2010)

20. S. V. Hainsworth, Polym. Test. 26, 60-70 (2007)

21. M. Flamm, J. Spreckels, T. Steinweger, U. Weltin, Int. J. Fatigue 33, 1189-1198 (2011)

22. J. B. Le Cam, B. Huneau, E. Verron, Int. J. Fatigue 52, 82-94 (2013)

23. I. Masquelier, $\mathrm{PhD}$ thesis, UBO, 2014

24. N. Saintier, G. Cailletaud, R. Piques, Int. J. Fatigue 28, 61-72 (2006)

25. B. Huneau, I. Masquelier, Y. Marco, O. Brzokewicz, P. Charrier, ECCMR VIII, 393-398 (2013) 